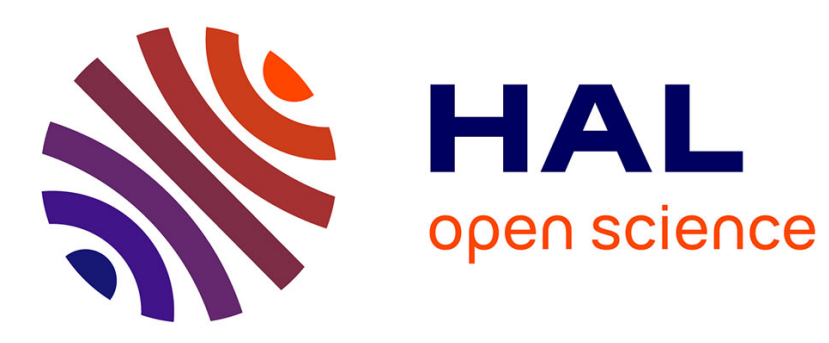

\title{
Tout dire ou le gouvernement de la langue Philippe Roussin
}

\section{To cite this version:}

Philippe Roussin. Tout dire ou le gouvernement de la langue. Communications, 2016, Démocratie et littérature. Expériences quotidiennes, espaces publics, régimes politiques, 99, pp.69-93. hal-03148088

\section{HAL Id: hal-03148088 \\ https://hal.science/hal-03148088}

Submitted on 11 Mar 2021

HAL is a multi-disciplinary open access archive for the deposit and dissemination of scientific research documents, whether they are published or not. The documents may come from teaching and research institutions in France or abroad, or from public or private research centers.
L'archive ouverte pluridisciplinaire HAL, est destinée au dépôt et à la diffusion de documents scientifiques de niveau recherche, publiés ou non, émanant des établissements d'enseignement et de recherche français ou étrangers, des laboratoires publics ou privés. 


\section{Tout dire ou le gouvernement de la langue}

\section{Philippe Roussin}

\section{Citer ce document / Cite this document :}

Roussin Philippe. Tout dire ou le gouvernement de la langue. In: Communications, 99, 2016. Démocratie et littérature. Expériences quotidiennes, espaces publics, régimes politiques. pp. 69-93;

https://www.persee.fr/doc/comm_0588-8018_2016_num_99_1_2809

Fichier pdf généré le 04/01/2019 


\title{
Resumen
}

Decir todo o el gobierno del lenguaje.

Decir todo es una expresión que aparece en la lengua francesa del siglo XVIII. Los contextos de uso y las inflexiones semánticas que ha conocido permiten comprender las modificaciones sucesivas de las fronteras entre la esfera privada y la esfera pública. Se analiza el periodo $1750-$ 1990 escogiendo tres momentos : nacimiento de la autobiografía y derecho de la crítica, hasta el periodo revolucionario en el siglo XVIII ; combates en torno a la libertad de prensa y la estética realista en el siglo XIX ; debates relacionados con el lugar de la literatura a partir de 1945 y hasta los años 1960, donde la expresión designará lo que parece ser la tarea de la literatura.

\begin{abstract}
Saying everything or the government of the tongue.

To say everything is an expression that appears in the French language in the 13th century. The contexts of use and semantic inflections it has known allow an understanding of the fluctuating boundaries between private and public spheres. An analysis of the 1750-1990 period isolates three moments : the birth of autobiography and the right to criticize, until the revolutionary period in the 18th century ; fights around the freedom of the press and realistic aesthetics in the 19th century ; debates about the place of literature from 1945 until the 1960s when the term comes to mean what appears to be the aim of literature.
\end{abstract}

\section{Résumé}

Tout dire est une expression qui apparaît dans la langue française au XIlle siècle. Les contextes d'usage et les inflexions sémantiques qu'elle a connues permettent de comprendre les modifications successives des frontières entre sphère privée et sphère publique. On analyse la période 1750-1990 en isolant trois moments : naissance de l'autobiographie et droit de la critique, jusqu'à la période révolutionnaire au XVIIle siècle ; combats autour de la liberté de la presse et de l'esthétique réaliste au XIXe siècle; débats concernant la place de la littérature à partir de 1945 et jusque dans les années 1960, où l'expression en vient à désigner ce qui semble être la tâche de la littérature. 
Philippe Roussin

\section{Tout dire ou le gouvernement de la langue}

La longue durée de l'existence attestée de l'expression tout dire dans la langue française nous met en mesure, à travers l'analyse des auteurs, des contextes d'usage et des inflexions sémantiques que la formule a connues, d'approcher les configurations successives de ce que nous nommons habituellement un «espace public ${ }^{1}$. Tout dire n'a jamais reçu de définition juridique et désigne plutôt un excédent ou un écart par rapport à la liberté d'expression juridiquement définie. Tout dire appartient, par ailleurs, si l'on veut, aux sphères de l'aveu et du franc-parler. Michel Foucault, qui a isolé ces deux catégories de discours, ne les a, sauf erreur, pas rapprochées. Quel est le point commun entre l'aveu de la confession chrétienne et la parrésia du citoyen de la démocratie et de la république?

L'interprétation la plus convenue de l'expression est sans doute celle que l'on trouvera dans le récit classique de l'histoire de la liberté d'expression selon le libéralisme. Plus fondamentalement, tout dire semble être ce lieu où la distinction des notions du privé et du public est susceptible de se brouiller et de se perdre, chaque sphère empruntant et devenant poreuse à l'autre. À partir du privé, une expression et une représentation privées se font reconnaître pour une représentation collective et passent ainsi les frontières de l'espace public; à l'inverse, l'injonction de tout dire - idéal de visibilité entière de la société ou phénomène d'ordre totalitaire - sera potentiellement lourde du risque de la disparition de l'espace privé : «si le droit de tout dire » est « la forme même de la liberté humaine », à l'inverse

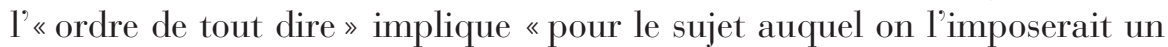
état d'esclavage absolu» ${ }^{2}$.

Dès le début, se pose la question de savoir à qui, à combien, dans les limites de quelle communauté et dans quelles circonstances l'on peut tout dire. Soit la question de l'amitié, qui est, depuis Aristote, une affaire politique et non privée: c'est l'amitié entre citoyens, dans la polis ${ }^{3}$. Guez de 
Balzac, au milieu du XVII ${ }^{\mathrm{e}}$ siècle, en prend la mesure: "Voici le privilège de la parfaite amitié. Elle donne la liberté de tout faire, de tout dire, de tout oser, sans qu'on doive craindre d'en être repris ${ }^{4}$. » L'amitié, on le sait, se décline au masculin - les noms des auteurs cités dans la suite de cet article le disent assez. Il s'agit de savoir, alors, si tout dire a un genre et s'il peut exister une mixité du tout dire.

Tout dire s'inscrit dans l'histoire de la négociation jamais achevée des déplacements de frontière entre privé et public, esthétique et droit, littérature et politique, dire et ne pas dire ${ }^{5}$. Les conditions de circulation de la parole sont ici centrales. Dans le républicanisme d'un Rousseau, opposé à la société mixte des salons où l'on pratique la conversation entre hommes et femmes, tout dire est d'abord une affaire d'hommes.

Rhétoriquement et esthétiquement, tout dire appartient à un horizon que l'on dira moderne, en ce sens qu'on peut y voir un renversement du tout est dit des anciens ( TTout est dit, et l'on vient trop tard depuis plus de sept mille ans qu'il y a des hommes » - La Bruyère, Caractères, 1696). L'expression apparaît dans la langue au XIII ${ }^{\mathrm{e}}$ siècle. La première occurrence attestée dans la littérature profane de langue française se trouve dans la Première Continuation de Perceval (composée vers 1205-1210), elle est donc contemporaine de l'institutionnalisation de la confession par l'Église (Canon 21, IVe concile de Latran, 1215). Les occurrences, ensuite, sont particulièrement concentrées dans les farces. Écrivant au temps des guerres de Religion, Montaigne est, sans doute, le premier auteur à faire sortir l'expression de son cadre d'origine pour la projeter dans l'espace public du livre et hors du secret du confessionnal. Dans ce «premier livre d'introspection profane»(Auerbach, Mimesis) que sont les Essais, il entend se «rendre public en connaissance» et déclare dire «vrai [... autant que je l'ose dire ». Il indique avoir « mes lois et ma court pour juger de moi » : « il n'y a que vous qui sçache si vous êtes lâche et cruel, ou loyal et dévotieux ( «Du repentir » ${ }^{6}$. Il doute que le repentir permette de désavouer les péchés les plus ancrés en nous et de s’en dédire. La véridicité est le point central du contrat qu'il passe avec le lecteur. La formule se trouve dans l'essai Sur quelques vers de Virgile, dont la confession est un thème central :

Au reste, je me suis ordonné d'oser dire tout ce que j'ose faire: et me déplaît des pensées mêmes impubliables. La pire de mes actions et conditions ne me semble pas si laide, comme je trouve laid et lâche de ne l'oser avouer. [...] Il faut voir son vice et l'étudier pour le redire : ceux qui le cèlent à autrui, le cèlent ordinairement à eux-mêmes $[. .$.$] En faveur des$ Huguenots, qui accusent notre confession privée et auriculaire, je me confesse en public, religieusement et purement. [...] Je suis affamé de me 
faire connaître : et ne me chaut à combien, pourvu que ce soit véritablement $^{7}$.

Montaigne se confesse en public et critique ailleurs sévèrement le mensonge. Il ouvre l'espace public en élargissant le cercle de l'amitié pour y inclure les femmes, alors que «ce sexe par nul exemple n'y est encore pu arriver et par le commun consentement des écoles anciennes en est rejeté ${ }^{8}$. C' est aux lectrices comme faisant partie du public que s'adresse le chapitre : «Je m'ennuie que mes Essais servent les dames de meuble commun seulement, et de meuble de sale: ce chapitre me fera du cabinet: J'aime leur commerce un peu privé : le public est sans faveur et saveur. » Et c'est à elles que Montaigne se permet de tout dire - il s'agit de la sexualité 9 .

La stabilité de l'expression au cours des siècles permet de repérer des moments de grande intensité. J'en distingue trois, regroupés, sans surprise, au sein de la période moderne, 1750-1990: les Lumières de la seconde moitié du XVIII ${ }^{\mathrm{e}}$ siècle et de la Révolution française, lorsque la formule est attachée à la naissance du genre autobiographique, au droit de la critique et à l'extension de la sphère publique; les combats autour de la liberté de la presse et de l'esthétique romanesque au XIXe siècle; les années 19451980 des débats sur le statut de la littérature au sortir de la Seconde Guerre mondiale et la nature de son rapport à la démocratie lorsque, dans la théorie de la littérature, l'énoncé en vient à désigner une sorte de droit de l'expression libre à l'infini et à se confondre, même, un temps avec une définition de la littérature - une prescription aussi : la tâche et la fonction de la littérature moderne, répète-t-on alors, seraient de tout dire.

Tout dire s'est constitué comme un énoncé stable avec les Lumières au point de devenir un quasi-topos de l'époque. Il y a alors, me semble-t-il, deux grandes manières de comprendre l'énoncé.

Il figure, on le sait, en bonne place dans les ébauches et au IVe livre des Confessions de Rousseau:

Je voudrais pouvoir en quelque façon rendre mon âme transparente aux yeux du lecteur, et pour cela je cherche à la lui montrer sous tous les points de vue, à l'éclairer par tous les jours [...] En lui détaillant avec simplicité tout ce qui m'est arrivé, tout ce que j’ai pensé, tout ce que j'ai senti, je ne puis l'induire en erreur [...] Ce n'est pas à moi de juger de l'importance des faits, je les dois tous dire [...] Je n'ai qu'une chose à craindre dans cette entreprise; ce n'est pas de trop dire ou de dire des mensonges; mais c'est de ne pas tout dire, et de taire des vérités ${ }^{10}$. 
Rousseau invente une nouvelle manière de lier le sujet, le langage et le public: il s'agit de «s'exprimer soi-même, face au plus large auditoire possible, afin de manifester une vérité inconnue»(Jean Starobinski). Impératif pour parvenir à l'identité entre auteur, narrateur et personnage, c'est-à-dire pour faire cö̈ncider la personne et le personnage social, l'engagement de tout dire est certainement celui de dire toute la vérité. Mais dans quel but et pour quoi faire? En fait, tout dire engage aussi les questions de la souffrance et de la présence déçue : on dit tout pour avouer (les fautes de l'enfance, l'abandon des enfants à l'âge adulte), se justifier, promettre, demander, être aimé, accuser - selon le déploiement des différents actes illocutoires possibles. Mais ce peut être aussi laisser libre cours à la peur, à la colère ou au ressentiment: Céline est, ici, un héritier en droite ligne de Rousseau. Parce qu'il souffre, Rousseau doit être écouté, et parce qu'il souffre, il est humain. Entre témoignage et visée apologétique du récit de vie, l'obligation de tout dire est centrale :

Dans l'entreprise que j'ai faite de me montrer tout entier au public, il faut que rien de moi ne lui reste obscur ou caché; il faut que je me tienne incessamment sous ses yeux, qu'il me suive dans tous les égarements de mon cœur, dans tous les recoins de ma vie; qu'il ne me perde pas de vue un seul instant, de peur que, trouvant dans mon récit la moindre lacune, le moindre vide, et se demandant, qu'a t-il fait durant ce temps-là, il ne m'accuse de n'avoir pas voulu tout dire ${ }^{11}$.

De la part de l'auteur des Confessions, tout dire revient à opter pour le langage de l'honnêteté contre celui de la décence. Ce choix doit être replacé dans le cadre de la réflexion sur le sort du langage et de la parole ( «la première institution sociale»-Essai sur l'origine des langues) et de la critique de la civilisation engagée par Rousseau, dès le Discours sur les sciences et les arts : conflit entre politesse (le « voile uniforme et perfide de la politesse») et sincérité (ou authenticité) ; dénonciation, politique et morale, des conventions sociales; débat sur la moralité et le progrès, qui oppose les philosophies anglaise et française, au XVIII ${ }^{\mathrm{e}}$ siècle. Je renvoie aux premières pages du Discours sur les sciences et les arts, où Rousseau associe peuples policés, politesse et esclavage : les « guirlandes de fleurs sur les chaînes de fer» vont avec le «langage apprêté ». La politesse uniformise : «sans cesse la politesse exige, la bienséance ordonne; sans cesse on suit des usages, jamais son propre génie. On n'ose plus paraître ce qu'on est » ${ }^{12}$.

Dans le républicanisme de Rousseau, la politesse est le signe d'une société corrompue: "Plus l'intérieur se corrompt et plus l'extérieur se compose» ( "Dernière réponse», Discours sur les sciences et les arts). Elle porte à leur paroxysme l'inauthenticité et la corruption des sociétés modernes, tandis 
que la conversation de la «société choisie » des salons interdit la sincérité et masque l'indifférence à la vérité. Elle n’est pas seulement hypocrisie mais aussi asservissement: la langue servile d'un sujet obéissant ${ }^{13}$. Tout dire signifie dès lors écrire en ayant rompu avec « le ton des sociétés, les matières qu' on y traite et la manière de les traiter ${ }^{14}$ », s'adresser à un public qui n'est pas une société, couper court à la conversation et mettre fin à la politesse, s'il est vrai que, comme le veulent manuels et traités, "la politesse consiste à ne rien faire et à ne rien dire qui puisse déplaire aux autres, à faire et à dire tout ce qui peut leur plaire et cela avec des manières et une façon de s'exprimer qui aient quelque chose de noble, d'aisé, de fin et de délicat ». La sincérité du tout dire est ce qui est à proscrire : "l'homme est naturellement sincère; il aime à dire ce qu’il pense, à témoigner ce qu'il sent. Cette disposition, quoique louable en elle-même, est un grand obstacle à la politesse, qui prescrit de ne rien faire et de ne rien dire qui puisse déplaire aux autres; d'où il s'ensuit qu'il ne faut pas dire tout ce qu'on pense, ni faire tout ce qu'on voudrait ${ }^{15}{ }^{»}$. Tout dire annonce la fin de la gestion rhétorique des rapports hiérarchiques de proximité et de distance entre les sujets, de même que l'autobiographie cherche à réduire à rien la distance entre le sentiment et l'expression. Cette nouvelle pratique d'écriture qui rend publique la vie privée ne va pas de soi. Car parler de soi, c'est aussi parler des autres ${ }^{16}$. Diderot condamne l'entreprise de divulgation abusive de vérités que l'on ne devrait pas connaître : Rousseau laisse «sur son tombeau la révélation des secrets qui lui ont été confiés ou qu'il a surpris de son vivant» (Essai sur les règnes de Claude et de Néron). Pour l'esthétique et la sociabilité du XVIII siècle, tout dire, à l'évidence, demeure une faute de goût - «Le secret d'ennuyer est celui de tout dire»(Voltaire, Discours en vers sur l'homme, 1738) - et, notamment, heurte la règle qui veut qu'il n'est pas bon de tout dire (par égard) au lecteur dont il s'agit de faire un témoin qui participe à la compréhension et à la conclusion du discours.

Jusqu'à lui, tout dire avait eu pour cadre l'amitié mais, dans les Confessions, Rousseau dénonce la nature de ce lien qui attache les grands et les hommes de lettres: "J'étais né pour l'amitié [...] Mais sitôt que j'eus un nom, je n'eus plus d'amis»(Confessions, livre VIII). Celui qui a montré son intériorité n'a obtenu, en retour, que la distance. L'écriture des Confessions est alors une réponse à la présence déçue, à l'amitié trahie (Diderot, Grimm, la coterie holbachique), à la publication du Sentiment des citoyens (1764), le pamphlet anonyme dans lequel Voltaire a fait part de l'abandon par Rousseau de ses enfants, autant qu'à la condamnation de l'Émile, à Paris et à Genève, en 1762. Du modèle religieux et moral de la confession - sincère et entière par principe - dont l'autobiographie hérite, il conserve l'impératif $\mathrm{d}^{\text {" }}$ absolue véracité ${ }^{17} »$. L'auteur des Confessions ne s'adresse plus à Dieu, dit-on souvent. En réalité, faute d'amis, il définit surtout, en 
disant tout, un public qui a cessé d'être une société: "Me voici donc seul sur la terre, n'ayant plus de frère, de prochain, d'ami ${ }^{18}$. . Le «souverain juge » devant lequel il se présente à la première page de son livre est l' « innombrable foule de mes semblables », l'auditoire universel : " le destinataire de sa parole est une collectivité illimitée»(Jean Starobinski). La publication sous les yeux du public détrône la lecture et la circulation restreinte des œuvres dans les limites de la société mondaine. Antoine Lilti écrit que la réévaluation du public, «si importante dans le nouveau langage littéraire et politique de la fin de l'Ancien Régime, est une des pierres de touche de la dénonciation de la sociabilité mondaine et du rôle qu'y jouent les hommes de lettres». Et il ajoute: «comme forme d'agrégation fondée sur les relations interpersonnelles et sur des pratiques de distinction, la société s'oppose très nettement au public, que celui-ci désigne les destinataires d'une œuvre ou l'ensemble du corps politique ${ }^{19}$.

Dans le néo-républicanisme qui remet au goût du jour les vertus des Anciens, tout dire est une vertu citoyenne, à l'opposé des manières des monarchies civilisées et des sociétés du luxe ${ }^{20}$. Le modèle républicain de l'espace public de Rousseau s'oppose au modèle du "processus économique de civilisation ${ }^{21} »$ comme aux doctrines de la sociabilité qui attribuent au « doux commerce» des effets civilisateurs sur les rapports sociaux. Il s'oppose au modèle whig de la commercial society et du marché qui inspire le modèle de l'espace public anglais. Depuis le texte célèbre de Joseph Addison sur la Bourse de Londres paru dans le Spectator en 1711, et repris par Voltaire dans les Lettres philosophiques (1733) ${ }^{22}$, le marché est devenu le modèle de l'échange des opinions. Les idées circulent comme les biens. La métaphore du commerce (des biens, des idées, entre les sexes et les personnes) est, là, omniprésente: la Bourse est le lieu où les confessions coexistent, le lieu de l'échange d'idées sans conflits, un espace de tolérance des différences et des opinions. Le commerce est «un lien d'union et d'amitié » entre les individus comme entre les nations (Adam Smith, La Richesse des nations).

Dans les histoires du progrès et de la civilisation des Lumières, l'histoire des femmes joue un rôle essentiel ${ }^{23}$. Elles sont une force civilisatrice dans l’histoire et dans la société contemporaine, associée à la modernité :

Le commerce continuel, si vif et si poli, des deux sexes a introduit en France une politesse assez ignorée ailleurs. La société dépend des femmes. Tous les peuples qui ont le malheur de les enfermer sont insociables $^{24}$.

C'est en jugeant de la nature et de la qualité de la relation entre les hommes et les femmes que Hume distingue entre civilisation et barbarie: la condition des femmes est la mesure du degré de développement d'une 
société. Dès 1742, Hume exhorte les femmes et les savants à se rapprocher afin de rendre la conversation moins frivole et la culture plus compréhensible et plus utile :

\begin{abstract}
Quelle meilleure école pour les mœurs que la compagnie de femmes vertueuses, où le mutuel effort de plaire polit insensiblement l'esprit, où l'exemple de la douceur et de la modestie de la femme se communique à ses admirateurs, et où la délicatesse de ce sexe met chacun en garde, de crainte de l'offenser en heurtant la décence ${ }^{25}$.
\end{abstract}

Dans les sociétés commerciales et dans les salons, les femmes entrent dans la sphère publique à parité avec les hommes. L'espace public de Rousseau est, lui, régi par le masculin : on peut tout y dire, pour cette raison. Dans le Discours sur les sciences et les arts, il condamne un « siècle devenu plus poli» et une société où il n’y a plus de citoyens. Il explique que la civilisation «effémine les courages ». Dans la Lettre à d'Alembert, il avance que la société mixte des salons a pour effet que «les femmes nous rendent femmes ». Il donne un sens masculin au concept de société politique considéré comme l'espace des citoyens politiquement actifs et en armes: «Les deux sexes doivent se rassembler parfois et vivre ordinairement séparés. » La prévalence de la politique républicaine dans son discours place les femmes hors de l'espace public: "Qu'un monarque gouverne des hommes ou des femmes, cela lui doit être assez indifférent, pourvu qu'il soit obéi; mais dans une République, il faut des hommes » 26.

L'usage du tout dire déborde largement le monde de l'intimité et de la sphère privée. L'expression, qui, dans la poétique classique, était réservée au genre de la satire, devient maintenant associée à celui de la critique ${ }^{27}$. Le partage entre les deux genres et les limites entre liberté et licence doivent toujours être rappelés: "Cette liberté de dire tout, d'écrire tout ce qui convient à la vérité, pour la maintenir dans ses droits, ne s'étend pas jusqu'à la licence, et n'autorise pas à composer des libelles ${ }^{28}$. » L'énoncé est fréquemment - sans réserves et de manière toujours positive - associé au bien du public, à la liberté de discussion et d'expression et à la critique. Il se confond avec le programme d'un élargissement de l'espace public et traduit, presque hyperboliquement, le «principe de publicité des débats qui doit régir la société des citoyens ${ }^{29}$ ». Les historiens ont montré que les philosophes des Lumières étaient loin de se rallier au principe de la liberté de la presse et qu'ils la concevaient plutôt comme «un idéal à défendre dans un monde réel d'intérêts économiques et de groupes de pression politiques $^{30} »$. Elle n'a jamais fait, de leur part, l'objet d'une campagne cohérente et c'est plutôt l'image complexe de complicités et de compromis entre 


\section{Philippe Roussin}

les différents acteurs (auteurs, censeurs, parlements, cours, administration royale, libraires) qui s'impose ${ }^{31}$. Les philosophes ont exprimé leurs idées les plus radicales en la matière quand ils ont été confrontés à la censure et à la répression, au moment de la condamnation de l'Encyclopédie en 1759 ; ils se sont ensuite montrés beaucoup plus mesurés. La position la plus libérale est celle de Diderot qui, dans sa Lettre sur le commerce des livres (1763, mais publiée seulement en 1863), plaide pour « les permissions tacites à l'infini », système moins règlementé que celui des privilèges ${ }^{32}$. Si les philosophes ne sont pas enthousiastes lorsqu'ils théorisent la liberté de la presse, c'est parce que l'idée d'un public autopolicé et d'une « opinion publique autorégulée »(Charles Walton) voisine chez eux avec le constat que le tribunal de l'opinion doit jouir d'une juridiction limitée : on est pour l'absolution de la censure préalable ou contre la répression de la police après publication, mais jamais en faveur des deux mesures à la fois.

Tout dire n'en symbolise pas moins l'idéal d'une pensée qui estime pouvoir rompre avec la double conscience, la politique de la prudence philosophique (Descartes: «j'avance masqué»), la dissimulation d'État (Gracián : "Conseil politique : ne se point ouvrir ni déclarer »), celle qui est favorable à la protection de la liberté de pensée dans une société coercitive (Cremonini : « À l'intérieur, fais comme il te plaît; à l'extérieur, range-toi à la coutume») ou avec la politique du silence (La Bruyère, «Des grands », Caractères : «L'on doit se taire sur les puissants »). La formule opère alors comme un retournement de la dissimulation baroque : pratique de gouvernement fondée sur la gestion du secret, pratique des dissidents religieux, mais aussi technique intégrée à la stratégie de l'opposition (qui permet de sauvegarder la liberté intérieure) et à son éthique (ce qui conduit, paradoxalement, à rapprocher dissimulation et honnêteté) ${ }^{33}$. La pensée peut s'exprimer plus ouvertement et rompre avec l'ésotérisme - précaution jusqu'alors de règle. Il est moins nécessaire d'écrire à couvert, entre les lignes, pour les seuls lecteurs attentifs. La diffusion des idées n’a plus nécessairement besoin de la clandestinité ni du déguisement. La possibilité de tout dire indique, pour l'art d'écrire, la sortie de l'âge de la persécution $^{34}$. Les références politiques et historiques vont alors à Rome, lorsque la revendication de la liberté d'expression se nourrit du souvenir des liens anciens entre liberté et éloquence (Helvétius : sous le règne de Trajan, «il était permis de tout dire, de tout penser, de tout écrire ${ }^{35}$ ») ou, sinon, à l'Angleterre contemporaine: "Pope a le droit de tout dire et moi de me taire» (Voltaire, Discours en vers sur l'homme).

La politique des Lumières met l'accent sur la diffusion des compétences critiques dans l'ensemble de la société. Dans cette acception, tout dire revient à poser la question de la diffusion des discussions politiques et coïncide avec les limites de l'espace public nécessaire à la vérité. La publi- 
cité des points de vue sert le bien commun, elle permet la constitution d'un public éclairé et participe au rétablissement de la figure du citoyen : «La nécessité où se trouve alors le citoyen de s'occuper d'objets importants, la liberté qu'il a de tout penser et de tout dire, donne plus de force et d'élévation à son âme; l'audace de son esprit passe dans son cœur ${ }^{36}$. » La diffusion de l'esprit d'enquête et la communication du savoir concourent au progrès des institutions politiques. Dans De l'homme, Helvétius consacre de nombreuses pages à faire valoir les avantages de la liberté de la presse : elle n'a rien de «contraire à l'intérêt général », elle est l' " aliment de l'émulation» et, plus généralement, "le moyen de découvrir la vérité que l’on doit aux peuples » 37 . Dans De l'esprit, brûlé sur les marches du palais de justice et dont la publication, en 1758, entraîne, avec la condamnation de l'Encyclopédie, la plus grave crise des Lumières, il évoque plus particulièrement la liberté de tout dire:

Au moment même qu'on interdirait la connaissance de certaines vérités, il ne serait plus permis d'en dire aucune. Mille gens puissants et souvent même mal intentionnés, sous prétexte qu'il est quelquefois sage de taire la vérité, la banniraient entièrement de l'univers. Aussi le public éclairé qui seul en connaît tout le prix la demande sans cesse [...]. Il sait combien il est utile de tout penser et de tout dire : et que les erreurs mêmes cessent d'être dangereuses, lorsqu'il est permis de les contredire. Alors elles sont bientôt reconnues pour erreurs; elles se déposent bientôt d'elles-mêmes dans les abîmes de l'oubli, et les vérités seules surnagent sur la vaste entendue des siècles ${ }^{38}$.

Tandis que la censure proscrivait la vérité, la liberté d'expression prévient l'erreur. La liberté d'expression est un moyen de la découverte de la vérité. Et l'extension des communications et des échanges entre ses membres profite à la société. L'énoncé signifie, dans tous les cas, que la liberté de pensée n'est rien sans la liberté de parole ${ }^{39}$.

$*$

En 1788, Mirabeau donne la première traduction française de l'Areopagitica, le grand manifeste pour la liberté de la presse publié par Milton, en 1644, au cœur de la Première Révolution anglaise. La Déclaration des droits de l'homme et du citoyen de l'été 1789 stipule, dans son article XI, que « la libre communication des pensées et des opinions est un des droits les plus précieux de l'Homme: tout Citoyen peut donc parler, écrire, imprimer librement, sauf à répondre de l'abus de cette liberté dans les cas déterminés par la Loi ». La Révolution échouera à définir ces abus, on le 


\section{Philippe Roussin}

voit dès janvier 1790, à l'occasion du premier plan d'organisation de la presse qui suit les discussions orageuses de décembre 1789 sur les libelles calomnieux visant les députés. Présenté par Sieyès, le "projet de loi contre les délits qui peuvent se commettre par la voie de l'impression » pose que la liberté de la presse doit avoir, comme toute liberté, «ses bornes légales». Il est jugé liberticide: «le mot livre défendu doit être supprimé de notre langue »(Loustalot, Révolutions de Paris, 23-30 janvier 1790. p. 126). Partisan intransigeant du contrôle populaire exercé sur la représentation nationale, Marat écrit aux lendemains de la discussion parlementaire du rapport de Sieyès : "Quant il est permis de tout dire, la vérité parle toujours et son triomphe est assuré [... La liberté de tout dire n’a d'ennemis que ceux qui veulent se réserver la liberté de tout faire. Oui, je ne crains pas de l'assurer, il n'est point d'opinions dangereuses, point d'opinions incendiaires tant qu'elles sont libres ${ }^{40}$. Les journalistes ont une interprétation extensive de l'article XI: il ne concernerait pas seulement l'abolition de la censure préalable mais couvrirait aussi celle de la répression après publication. Il est d'autant plus impossible de légiférer sur la calomnie que la presse remplit une double fonction : la publicité des débats politiques et le contrôle de la représentation nationale; elle est un espace de liberté et une forme de censure publique ${ }^{41}$. Pendant une courte période, à l'été 1791, émerge chez les Jacobins une position qualifiée de «libertaire» (libertarian) par Charles Walton. Lanthenas, médecin, député girondin - qui votera contre la mise en accusation de Marat le 14 avril 1793 et que Marat sauvera, ensuite, lors de la proscription des Girondins en juin de la même année - explique alors :

Plus nous irons et plus la publicité en tout, et la facilité de tout connaître, nous accoutumeront à tout voir, à tout entendre, à tout dire, à tout exprimer, à tout examiner, à tout discuter, à n'être étonné de rien, à ne juger rien sur parole, à n'avoir en un mot ni idolâtrie ni superstition $[\ldots]$ Si nous voulons sauver la chose publique, soyons calmes, réfléchis, écoutons tout; laissons tout dire [...] Que la loi punisse les actions seules.

Il propose d' "abroger toutes les anciennes lois » relatives aux injures, aux calomnies, aux blasphèmes, aux cultes religieux, aux théâtres, à la presse, et de les remplacer par « des règlements qui consacrent l'indépendance la plus illimitée de la communication des pensées et en favorisent le développement » ${ }^{42}$. Minoritaire, cette position libertaire disparaît à mesure que l'on approche de la Terreur et qu'une définition intolérante et floue de l'ennemi se diffuse dans les lois criminelles.

En Angleterre, le penseur radical qu'est William Godwin est alors le plus proche de ces positions libertaires. Dans An Enquiry Concerning Poli- 
tical Justice (1793), il se demande si les institutions politiques (le gouvernement), qui influencent fortement la formation des opinions, ont une quelconque justification et il envisage leur suppression. Il propose de simplifier les relations sociales, de sorte que les hommes se parlent sur un pied d'égalité - «un homme parlant à un homme». La communication sans limites des opinions concernant le gouvernement est, selon lui, indispensable à la vie d'une société libre. La sincérité doit être la règle de l'engagement du citoyen dans les affaires civiques et dans la vie de la communauté, lui permettant de prendre la parole à partir d'une déclaration strictement privée jusqu'à l'action dans l'intérêt général :

Ce que je sais de la vérité, des mœurs de la religion, du gouvernement, il me faut le communiquer [...] Je suis tenu de traiter chaque homme avec une égale franchise [...] On a justement fait remarquer que la pratique papiste de la confession auriculaire avait produit quelques effets salutaires. Tout irait mieux si, au lieu d'une pratique ambiguë, et qui peut être convertie en une dangereuse machine de despotisme ecclésiastique, tout homme faisait du monde son confessionnal et de l'espèce humaine le gardien de sa conscience ${ }^{43}$.

Les lois tendant à supprimer les libelles diffamatoires sont, en réalité, des lois visant à limiter la pratique civique de la sincérité; or il est nécessaire de dire la vérité :

Le courage consiste en cette circonstance plus que dans n'importe quelle autre dans l'audace de parler de tout ce dont la profération peut conduire au bien [the daring to speak everything, the uttering of which may conduce to good] [...] L'économie vertueuse de la parole est notre affaire perpétuelle. N'importe quel moraliste est capable de nous dire que la moralité consiste, de façon éminente, dans le gouvernement de la langue [the government of the tongue]. Mais cette branche de la morale a longtemps été sens dessus dessous. Au lieu de nous enseigner ce que nous aurons à dire, on nous a appris à considérer ce que nous aurions à dissimuler ${ }^{44}$.

Il faut, enfin, faire droit à l'usage que Sade, rendu à la liberté en avril 1790, fait de l'expression dans ses écrits, au cours de la Révolution. On trouve l'énoncé, présenté comme un droit, dans «Français, encore un effort si vous voulez être républicains », appel public inséré dans La Philosophie dans le boudoir (1795) : "N'avons-nous pas acquis le droit de tout dire? Développons aux hommes de grandes vérités: ils les attendent de nous; il est temps que l'erreur disparaisse, il faut que son bandeau tombe à côté de celui des rois ${ }^{45}$. » La dernière page de l'Histoire de Juliette ou les 


\section{Philippe Roussin}

Prospérités du vice, publié en 1797, met en scène le basculement de l'âge de la dissimulation vers celui de la publicité. Au personnage de Clairwill, qui a rappelé la règle qui veut que l'aventure libertine se vive et ne s'écrive pas ( «Il faut tout faire et ne jamais tout dire»), répond la déclaration de Juliette, en une formule qui sera remise au goût du jour dans les années 1960 : "Pourquoi donc craindre de le publier, dit Juliette, quand la vérité même arrache les secrets de la nature, à quelque point qu'en frémissent les hommes? La philosophie doit tout dire ${ }^{46}$. " Dans la brève Idée sur les romans, qui accompagne Les Crimes de l'amour en 1799, Sade répond à la prolixité de Restif, qui s’en est pris à Justine ( DDanton le lisait pour s'exciter») et autres « ouvrages infâmes»du «monstre » Sade, «vil noble, méchant comme tous ses pareils, contre l'espèce humaine » : "On n'a jamais le droit de mal dire, quand on peut dire tout ce qu' on veut ${ }^{47}$. »

L'article 8 de la loi de 1819, qui oppose Constant et Bonald lors de sa discussion parlementaire, introduit le délit d'outrage à la morale publique, religieuse et aux bonnes mours. La tension entre dire et faire est, ensuite, au centre des combats pour la liberté de la presse qui opposent libéraux et ultras au XIX ${ }^{\mathrm{e}}$ siècle. Deux conceptions de la presse (et du langage) s'affrontent: l'une, qui y voit l'organe de l'opinion et insiste sur son rôle dans la construction d'un espace public; l'autre, qui y voit, souvenir de la période révolutionnaire, le départ de l'émeute dans le peuple et la cause de l'action. Deux ans après la loi de 1835, l'auteur du Traité des délits et contraventions de la parole écrit : «La parole [...] n'est autre chose qu'une action. À ce titre, parler c'est agir ${ }^{48} »$ - rappel explicite de la maxime « Scribere est agere».

Les libéraux, partisans de la liberté de la presse, n’ont d'autres ressources que de chercher à découpler la parole de l'action, pour convaincre que la parole est opinion et que l'opinion n'est pas un délit (rendu possible par la loi de 1819 et créé par la loi de 1822). Émile de Girardin, dont le journal, comme une douzaine d'autres titres, est temporairement interdit après les journées de juin 1848, en vertu de l'état de siège, reprend des arguments déjà exposés par Constant: la presse ne favorise pas l'émeute; au contraire, elle sublime les passions démocratiques. Il oppose au « droit ancien » le droit nouveau, le droit ancien étant le droit d'insurrection (complots, attroupements, émeutes, barricades, révolutions), le droit nouveau le droit de discussion (livres, brochures, journaux, écoles, clubs, banquets): «Nous sommes les ennemis déclarés du droit d'insurrection [...] nous sommes les partisans systématiques du droit de discussion le plus étendu. » Le droit nouveau - celui de « tout dire » - doit se substituer au droit ancien : 
« aucune limite légale ne doit être mise à la liberté de tout dire. La parole, comme la pensée, échappe à la loi positive » ${ }^{49}$. La presse n’est fugitivement libre qu'en 1830 et lors du "printemps des journaux », en 1848. Jusqu'à la grande loi républicaine de 1881, fruit d'une négociation entre républicains, monarchistes et catholiques, qui éteint les différentes formes de censure, l'autonomie du dire par rapport au faire n'est pas assurée.

Au cours du siècle, tout dire s’impose, parallèlement, comme un idéal et un horizon esthétiques pour les écrits qui relèvent de la Littérature, cette institution nouvelle qui succède aux Belles-Lettres. C'est d'abord la demande romantique d'une liberté dans le choix des sujets et du style: «nous voudrions un vers libre franc, loyal, osant tout dire » (Victor Hugo, préface de Cromwell, 1827). Tout dire s'impose, parce qu'il n'y a plus « ni règles ni méthodes » et que la littérature s'éloigne du discours, du bien dire, des hiérarchies esthétiques entre les genres et entre les formes. Soit, alors que «tous les mots n'avaient pas droit à la langue» dans l'ancien régime littéraire, le choix du «mot propre»contre la «périphrase» (Hugo). Contre l'idéal classique de la séparation des styles, tout dire revient à faire du laid une source d'imitation à côté du beau, du grotesque un élément d'art à côté du sublime, à « retrouver le Verbe dans l'argot»(Hugo): sa possibilité va de pair avec l'idée de la vitalité d'une langue non fixée. Cela n'est possible qu'au nom du génie, conformément à la nouvelle valorisation de l'originalité affranchie de toute tutelle. "La démocratie, écrit toujours Hugo, est dans cette littérature », où l'on entend l'écho des voix de Danton et de Robespierre. Il semble, cependant, que tout dire relève d'abord du domaine de la prose : "la poésie ne consiste pas à tout dire mais à tout faire rêver ${ }^{50}$ ».

En 1862, Hugo, encore lui, affirme, à propos des Misérables, que son « livre est fait pour tout dire » et, se référant à l'imaginaire de l'opacité et de la justice sociales du roman, il exhorte ainsi : "ne cachez rien, dites tout »51. Balzac, dans l'avant-propos de 1842 à La Comédie humaine, avait déjà expliqué qu'il entendait «copi[er] toute la Société ». L'énoncé devient un article clé des poétiques réalistes et naturalistes: "tout voir, tout savoir, tout dire»(Zola, Nouveaux Contes à Ninon, 1874). La formule a valeur de manifeste pour le genre romanesque, dépourvu de poétique mais dominant. Elle marque le souci d'exhaustivité et l'ambition de systématicité du réalisme, qui se veut connaissance complète de la réalité. Elle expose, avec la référence scientifique, la certitude positiviste d'un monde entièrement lisible et déchiffrable. La littérature romanesque est pensée comme un lieu de vérité sinon de dévoilement, ce qui existe selon l'hypothèse du dit et du non-dit. Le roman lui-même est une manière de totalisation. L'œuvre réaliste se donne pour un tout est dit. «Tout dire, tout connaître, tout guérir » (Zola, Le Docteur Pascal, 1893, conclusion du cycle des Rougon-Macquart). Elle dit aussi l'égalité nouvelle de tous les 
sujets en littérature: «tout a droit de cité en poésie» (Hugo, préface de Cromwell) ; "Nous disons tout, nous ne faisons plus un choix, nous n'idéalisons pas, et c'est pourquoi on nous accuse de nous plaire dans l'ordure » (Zola, Le Naturalisme au théâtre, 1881).

Il s'agit là d'un horizon lointain dont la loi et la critique, chacune à sa manière et avec ses moyens, rappellent l'inatteignable. En 1857, année des procès de Madame Bovary et des Fleurs du mal, l'expression est en faveur: "C'est la grande erreur du "réalisme"; il prétend être vrai parce qu'il dit tout $^{52}$. » Le substitut Pinard s'adresse ainsi aux jurés lors du réquisitoire du procès des Fleurs du mal : "Réagissez par un jugement [...] contre cette tendance malsaine qui porte à tout peindre, à tout décrire, à tout dire, comme si le délit d'offense à la morale publique était abrogé, et comme si cette morale n'existait pas ${ }^{53}$. »

Mais tout dire ne définit pas seulement une poétique. La liberté de parole serait un droit purement fictif si celui qui désirait l'exercer dépendait de l'autorité qu'il entend mettre en cause. Chez Zola encore, tout dire est gagé sur l'autonomie économique et sociale gagnée de haute lutte par le romancier via la conquête du marché et du public : "L’argent [...] l'a délivré de toute protection humiliante [et] a fait de l'ancien bateleur de cour, de l'ancien bouffon d'antichambre, un citoyen libre [...] Avec l'argent, il a osé tout dire $[\ldots]$ L'argent a créé les lettres modernes $[. .$.$] L'argent est notre$ courage et notre dignité, à nous écrivains, qui avons besoin d'être libres pour tout dire ${ }^{54}$. » Dans la République enfin stabilisée en 1877, Zola comprend la liberté de tout dire comme le fait de celui qui peut s'adresser au public sans en être le représentant: «Dans les partis politiques, il y a ce qu'on appelle la discipline [... Dans les lettres, heureusement, la discipline ne saurait exister. » À l'écrivain «que ses conditions d'existence ne forcent pas à la discipline», il « est permis, en un mot, d'être seul de son avis, parce qu'il ne fait pas corps avec un groupe et qu'il peut tout dire ${ }^{55}$. Cette possibilité de s'adresser à l'opinion définit la figure nouvelle de l'intellectuel à la fin du XIX ${ }^{\mathrm{e}}$ siècle.

Que devient la revendication de tout dire dès lors que le régime républicain fait droit à la liberté d'expression en 1881 (à l'exception des années 1892-1894, celles des attentats anarchistes et des «lois scélérates») et éteint, de fait, cette revendication? En 1924, dans le premier Manifeste surréaliste, Breton estime « dommage que les délits de presse ne soient plus guère réprimés». La question se déplace sur le seul terrain de la littérature, où elle se divise en, d'une part, une réflexion sur la place de celle-ci, à côté des autres discours, tous égaux en droit en régime démo- 
cratique et, d'autre part, une revendication de liberté absolue, soutenue par une conception aristocratique de la liberté comme jouissance d'un privilège. Tout dire engage alors l'histoire difficile de la désacralisation et de la démocratisation (de la pensée) de la littérature. J'en viens ainsi aux débats d'après 1945, en France.

Le recueil de poèmes de Baudelaire, condamné en août 1857 pour "atteinte à la morale publique et aux bonnes mœurs », est réhabilité le 31 mai 1949. La Société des gens de lettres obtient l'annulation du jugement, après quatre-vingt-douze ans de purgatoire. En 1958, l'œuvre de Sade se trouve, pour la première fois, publiquement disponible, à l'issue du procès gagné par son éditeur, Jean-Jacques Pauvert, contre les poursuites engagées en 1956. L'avocat général Boucheron a convaincu le jury qu’un écrivain devait pouvoir bénéficier d'une «franchise » littéraire ${ }^{56}$. La loi de 1881 ayant aboli les délits d'opinion et l'outrage à la morale publique et religieuse, l'œuvre de Sade, explique l'avocat général, ne peut être poursuivie sur la base de textes antérieurs abrogés. Il propose d'étendre aux écrivains la théorie de la franchise, déjà appliquée aux magistrats et aux témoins, «sous la forme d'une exemption exceptionnelle de responsabilité et sous le nom de franchise littéraire »; cette franchise est une dispense, non une immunité ${ }^{57}$. L'écrivain peut tout dire : ce qu'il dit reste détaché de la loi, de la vérité, du pouvoir.

En 1948, dans Qu'est-ce que la littérature?, revenant sur la littérature de la Résistance et sur les choix des hommes de lettres, collaborateurs ou non, au cours des quatre années de l'Occupation, Sartre, qui passe de préoccupations esthétiques à des préoccupations morales et politiques, revient sur l'autonomie du dire par rapport au faire: «La parole est un certain moment particulier de l'action et ne se comprend pas en dehors d'elle [...] parler c'est agir ${ }^{58}$. » Il invoque la torture, pratiquée dans le Paris de l'Occupation : "La littérature n'est pas un champ innocent et facile qui s'accompagne de tous les régimes. » Il théorise la responsabilité historique et morale de l'écrivain ${ }^{59}$. Je rappelle les formules célèbres : «La liberté d'écrire implique la liberté du citoyen. On n'écrit pas pour des esclaves. L'art de la prose est solidaire du seul régime où la prose garde un sens : la démocratie. »L'écrivain, homme libre, s'adresse à la liberté des lecteurs. En d'autres termes, sa liberté n'est rien sans celle du lecteur : «il ne suffit pas d'accorder à l'écrivain la liberté de tout dire, conclut-il, il faut qu'il écrive pour un public qui ait la liberté de tout changer». L'écrivain s'adresse par principe "à tous les hommes». Dans une allusion claire aux thèses surréalistes sur la négation de l'utile et à celles de Georges Bataille sur la littérature comme dépense somptuaire, il écrit qu’il s'agit de " remplacer la dépense par le don», de "renoncer au vieux mensonge 
aristocratique » et de «lancer à travers toutes nos œuvres un appel démocratique à l'ensemble de la collectivité » ${ }^{60}$.

Maurice Blanchot s'efforce de proposer une construction alternative à la théorie de la littérature engagée depuis Qu'est-ce que la littérature?. Sade, l' «écrivain par excellence», y joue un rôle central. L'invocation de son œuvre est une échappée hors du «moralisme national» (Gisèle Sapiro) qui a distingué l'atmosphère de la Libération. Plus fondamentalement, il semble, comme l'écrit Blanchot, qu'il n'y ait pas de leçon à tirer de la guerre récente. Ce n'est pas la Libération mais la Révolution et la Terreur qu'il choisit d'invoquer comme origine de la littérature (voir «La littérature et le droit à la mort », La Part du feu, 1949). Deux scènes primitives de la littérature s'affrontent: la torture sous l'Occupation, la Terreur révolutionnaire. Le nom de Sade permet de faire naître la littérature dans la Révolution et la Terreur. Blanchot peut alors prendre le contre-pied des positions de Sartre et assimiler responsabilité et limitation de la liberté: «La littérature n'a peut-être pas le droit de se tenir pour illégitime. Mais la question qu'elle renferme ne concerne pas, à proprement parler, sa valeur ou son droit ${ }^{61}$. » Elle n'a pas, non plus, en tout cas, de rapport avec l'action: «L'influence de l'écrivain est liée à ce privilège d'être maître de tout [...] la vérité c'est qu'il ruine l'action, non parce qu'il dispose de l'irréel, mais parce qu’il met à notre disposition toute la réalité 62 . »

Paradoxalement, c'est au moment où la liberté de tout dire n'est plus un enjeu de droit qu'elle va devenir un enjeu proprement esthétique et idéologique. Au cours des années 1960, la formule vaut quasi-définition de la littérature, pour la théorie et la critique de la période:

C'est bien la vérité de Sade: une vérité d'autant plus dangereuse qu'elle est claire, lucidement proposée, simplement exprimée - précisément à la dernière page des Prospérités du vice et sous la forme la plus lisible: «à quelque point qu'en frémissent les hommes, la philosophie doit tout dire». Tout dire. Cette seule ligne eût suffi à le rendre suspect, ce projet à le faire condamner, sa réalisation à le faire enfermer. Et il n'y a pas à en rendre responsable le seul Bonaparte. Toujours nous vivons sous un Premier consul, et toujours Sade est poursuivi à cause de la même exigence: tout dire; tout dire; il faut tout dire, la liberté est la liberté de tout dire, ce mouvement illimité qui est la tentation de la raison, son vœu secret, sa folie ${ }^{63}$.

Maurice Blanchot, l'auteur de ces lignes, insiste sur la liberté et sur l'individualisme radical de Sade, un anti-Rousseau (celui de la loi comme expression de la volonté générale) et un anti-Kant («la liberté n’est pas 
en dehors de la loi »-Fondements de la métaphysique des møurs). Il oppose la «puissance»que l'homme de Sade revendique à la loi : la "puissance n'a rien à redouter de la loi dont elle ne reconnaît pas la légitimité ${ }^{64}$ ».

Durant cette période, la littérature se trouve définie, à partir de la poétique, comme une pratique linguistique spécifique. La référence au langage permet de l'objectiver, identifiée à une réalisation maximale de ses possibilités. Elle est l'expérience qui met en ouvre l'infini du langage. Cet effort pour définir sa spécificité n’empêche pas une absolutisation de la littérature. Dire qu'elle peut tout dire, c'est dire qu'elle échappe à l'ordre du discours, à ses règles. Elle est étrangère au partage du langage commun et du langage privé, de l'intransitif et du transitif. Communication hors norme, la littérature est une contre-rhétorique, une critique de la langue et une dénonciation de l'ordre social du discours. Elle se confond avec l'expression affranchie des contraintes des discours et de l'idéologie. L'œuvre «figure la possibilité de tout dire et se définit paradoxalement comme la présentation d'une adresse générale » : «elle figure la possibilité de passer les limites que les institutions linguistiques et sociales de la réalité mettent paradoxalement à cette adresse » ${ }^{65}$. Cela revient à définir le geste d'écrire comme un geste quasi souverain, puisqu'on le donne pour capable de passer les limites du discours. Le geste souverain que constitue l'œuvre de Sade se trouve intégré dans une théorie de la transgression et de la souveraineté (Georges Bataille) : "La liberté souveraine, absolue, fut envisagée - dans la littérature - après la négation révolutionnaire du principe de la royauté ${ }^{66}$. » Le littéraire est exercice de souveraineté et non d'autonomie: "Le centre du monde sadique, c'est l'exigence de la souveraineté s'affirmant par une immense négation. » Tout dire revient alors à définir la littérature à partir d'un statut d'exception - la Terreur révolutionnaire, hors d'un État de droit.

Le langage à l'infini de la littérature est une récusation du langage commun. Il y a là un paradoxe du littéraire dans l'espace public et démocratique. La liberté est devenue pouvoir de tout dire, capacité à différencier et à marquer le rang. Pragmatiquement, tout dire est une asymétrie de la parole, sans réplique et sans interlocuteur ${ }^{67}$ : «Le langage n'a son lieu que dans la souveraineté solitaire du "je parle", rien ne peut le limiter en droit - ni celui auquel il s'adresse, ni la vérité de ce qu'il dit, ni les valeurs ou les systèmes représentatifs qu'il utilise ${ }^{68}$. » Le pouvoir de tout dire permet d'assigner du sens de manière libre et systématique : la littérature serait cette parole qui dispose du « pouvoir de modifier souverainement les valeurs et les significations de la langue ${ }^{69} »$.

En 1993, passé la guerre froide, dans Passions, Jacques Derrida veut rapprocher les positions de Sartre (le lien entre littérature et démocratie) 


\section{Philippe Roussin}

et de Blanchot (la liberté absolue de tout dire). Après d'autres philosophes du politique - Cornelius Castoriadis, Claude Lefort -, il pose alors une analogie structurale entre la littérature et la démocratie et introduit ainsi le droit de tout dire :

[la littérature] s'inscrit dans des conventions et des institutions qui [...] lui assurent en principe le droit de tout dire. La littérature lie ainsi son destin à une certaine non-censure, à l'espace de la liberté démocratique (liberté de la presse, liberté d'opinion, etc.). Pas de démocratie sans littérature, pas de littérature sans démocratie [...] La possibilité de la littérature, l'autorisation qu'une société lui accorde [...] tout cela va de pair politiquement avec le droit illimité de poser toutes les questions [...] d'analyser toutes les présuppositions, fussent-elles celles de l'éthique ou de la politique de la responsabilité.

Derrida conclut pourtant à l'impossibilité de réconcilier démocratie et droit de tout dire :

[l'] autorisation de tout dire constitue paradoxalement l'auteur en auteur non responsable devant quiconque [...] Cette autorisation de tout dire (qui va pourtant de pair avec la démocratie comme hyperresponsabilisation apparente du «sujet») reconnaitt un droit à la nonréponse absolue. [...] Il y a là une conception hyperbolique de la démocratie qui semble contredire un certain concept déterminé et historiquement limité de ladite démocratie, celui qui la lie au concept de sujet $[\ldots]$ responsable et devant $[\ldots]$ dire la vérité ${ }^{70}$.

La clause d'irresponsabilité invoquée ici est la suivante: l'écriture n'obéirait pas et s'affranchirait de la nature contractuelle des speech acts théorisés par Austin et par Searle. La littérature peut tout dire parce que, comme le répète la déconstruction, elle n'est pas performative.

Que la littérature ait pour tâche de tout dire, cette thèse persistera ${ }^{71}$. Cependant, l'histoire a une fin, peut-être seulement provisoire. La littérature a fini de se reconnaître dans les systèmes de totalisation romanesque. La Vie mode d'emploi a, ainsi, pour but de saisir, décrire et épuiser «non la totalité du monde - projet que son seul énoncé suffit à ruiner - mais un fragment constitué de celui-ci ${ }^{72}$ ». Barthes évoque le «monstre de la totalité »: « la Totalité tout à la fois fait rire et fait peur » ${ }^{73}$. Il définit la littérature comme une somme de savoirs, une mathesis, mais il fait remarquer qu'elle ne peut excéder le savoir de son époque et «ne peut tout dire ${ }^{74}$ ». 
Les définitions de la littérature livrées par les théories et la critique des cinquante dernières années ne correspondent pas aux leçons des pragmatiques actuelles. La littérature ne peut plus être approchée comme cet ensemble qui s'opposerait aux autres discours et en ferait la critique. Elle est le regroupement des énonciations singulières et des représentations privées qui se donnent pour des représentations publiques. Elle est un discours sans privilège, à égalité avec les autres discours qui s'analyse, positivement, en termes d'invention plutôt qu'à partir de ce qui est empêché de parler : «La vraie censure», poursuit Barthes, «ne consiste pas à interdire » mais «à étouffer, à engluer dans les stéréotypes » : « la censure sociale n'est pas là où on empêche, mais là où l'on contraint de parler » ${ }^{75}$. Il suffit de rappeler le projet du Dictionnaire des idées reçues tel que le décrit Flaubert : «Ce serait la glorification historique de tout ce qu'on approuve [...] On y trouverait donc, par ordre alphabétique, sur tous les sujets possibles, tout ce qu'il faut dire en société pour être un homme convenable et aimable ${ }^{76}$. » Parce que la littérature avait été ce domaine où l' "opacité sociale » s'était trouvée «représentée » et «dramatisée», Barthes suggérait de considérer l'écriture comme une pratique qui essaie de «périmer l'opposition du caché et du non-caché » et "non pas de tout dire » ${ }^{77}$ : «L'écriture n'est pas de l'ordre du "tout dire" [... Elle n'a pas à charge de dire quelque chose $^{78}$. » Contre les thèses de l'intransitivité, il rappelait le modèle contractuel de la langue. Dans un texte consacré à l'épistémologie de Saussure, il écrivait:

La langue n'est pas prise dans un procès de filiation, l'héritage y est dévalorisé $[\ldots]$ l'espace du mot n'est pas celui d'une ascendance ou d'une descendance, c'est celui d'une collatéralité: les éléments de la langue - ses individus - ne sont plus fils, mais concitoyens les uns des autres : la langue, dans son devenir même, n'est plus une seigneurie, mais une démocratie : les droits et les devoirs des mots (qui forment en somme leur sens) sont limités par la coexistence, la collaboration d'individus égaux [... Le modèle de la linguistique saussurienne, c'est la démocratie ${ }^{79}$.

Tout dire, au XVIII ${ }^{\mathrm{e}}$ siècle, est affirmation de la liberté du singulier et dessin de l'espace public où cette liberté doit œuvrer. Le libéralisme transpose dans le domaine public la diversité des opinions et des expressions privées. Lorsque cette transposition a lieu, à la fin du XIX ${ }^{\mathrm{e}}$ siècle, elle n'a pas pour autant résolu la question qui demeure dans l'ombre de la victoire du tout dire : celle de l'isègoria démocratique, de l'égalité d'accès à la parole publique ${ }^{80}$. Tout dire ne peut être seulement l'émancipation d'une autorité particulière qui s'arrogerait le pouvoir de décider de ce qui affecte la destinée et les représentations communes. C'est pourquoi tout dire se 


\title{
Philippe Roussin
}

heurte à la question de l'égalité discursive (isègoria) : aucun agent ne peut s'attribuer à lui-même la représentation de la totalité. En ce sens, tout dire - privilège, distribution du sens ou source éminente de la signification devient nécessairement problématique au regard de l'idée même d'espace public et de l’indétermination de la démocratie.

\author{
Philippe Roussin \\ roussin@ehess.fr
}

CNRS, Maison française d'Oxford

\section{NOTES}

1. Je remercie Luc Boltanski, Richard Scholar, Silvia Sebastiani, Anne Simonin, Helen Swift, Kate Tunstall et Sebastian Veg des discussions critiques qui ont accompagné la rédaction de cet article.

2. Piera Aulagnier, «Le droit au secret: condition pour pouvoir penser», in Piera Aulagnier (dir.), La Pensée interdite, Paris, PUF, 2009, p. 15. L'auteur, psychanalyste, discute le texte de Maurice Blanchot sur Sade dont il est question aux pages 84-85 de cet article.

3. Jacques Derrida, Politiques de l'amitié, Paris, Galilée, 1994.

4. Guez de Balzac, lettre du 15 janvier 1652, in Lettres de M. de Balzac à M. Conrart, 1659, p. 148.

5. Il existe à partir de Rome une licence qui serait d'un ordre strictement poétique et stylistique : «La licence poétique n'est pas du ressort de la juridiction des Censeurs [...] le pouvoir que les poètes ont de tout dire et de tout entreprendre, ne reçoit point de restriction [...] tout ce qui paraît irrégularité ou extravagance n'est qu'un pur effet de cette fureur que l'on qualifie d'enthousiasme et qui n'est pas sujette aux règles ou aux caprices de la raison humaine» (Adrien Baillet à propos de A. Ceba, auteur de La Regina Esther [1617], poème héroïque censuré par un archevêque de Palerme, Jugements des savants sur les principaux ouvrages des auteurs: t. V, édition revue, corrigée et augmentée par M. de la Monnoye, Suite des poètes modernes, Paris, 1722, p. 77).

6. Montaigne, «Du repentir», in Essais, III, II, Paris, Gallimard, coll. «Bibliothèque de la Pléiade », 1946, p. 780-782.

7. Montaigne, «Sur quelques vers de Virgile», in Essais, III, v, op. cit., p. 818-820.

8. Montaigne, «De l'amitié », in Essais, I, xxVIII, op. cit., p. 196. Voir le chapitre «Of FreeThinking and Friendship» dans Richard Scholar, Montaigne and the Art of Free-Thinking, Oxford, Peter Lang, 2010.

9. Montaigne, "Sur quelques vers de Virgile», op. cit., p. 820-821: "Mais venons à mon thème. Qu'a fait l'action génitale aux hommes, si naturelle, si nécessaire et si juste, pour n'en oser parler sans vergogne et pour l'exclure des propos sérieux et réglés? Nous prononçons hardiment: tuer, dérober, trahir; et cela, nous n'oserions qu'entre les dents? Est-ce à dire, que moins nous en exhalons en parole, d'autant nous avons loi d'en grossir la pensée? Car il est bon, que les mots qui sont le moins en usage, moins écrits, et mieux tus, sont les mieux sus, et plus généralement connus. [...] Ils s'impriment en chacun, sans être exprimez, et sans voix et sans figure. Il est bon aussi que c'est une action que nous avons mis en la franchise du silence.»

10. Jean-Jacques Rousseau, Confessions, in Euvres complètes, t. I, Paris, Gallimard, coll. «Bibliothèque de la Pléiade », 1959, p. 175.

11. Ibid., p. 59-60.

12. Jean-Jacques Rousseau, Discours sur les sciences et les arts, Paris, Gallimard, coll. «Folio», 2011, p. 32. 


\section{Tout dire ou le gouvernement de la langue}

13. Philippe Raynaud, La Politesse des Lumières. Les lois, les mœurs, les manières, Paris. Gallimard, 2013. Sur la conversation comme modèle des échanges dans la nouvelle société commerciale anglaise, voir surtout l'ouvrage de John Mee, Conversable Worlds, Literature, Contention and Community (1762 to 1830) Oxford, Oxford University Press, 2011.

14. Jean-Jacques Rousseau, "Mon portrait», in Euvres complètes, t. I, op. cit., p. 1124.

15. Abbé Nicolas Charles Joseph Truchet, «De la politesse», Essais sur divers sujets de littérature et de morale, tome second, 1749, p. 141-178; cf. p. 158: «La politesse est dans un sens plus importante que les qualités du cœur. On peut absolument se passer d'amis et d'amitié; mais on ne peut se passer de société; et il n'y a point de société sans politesse. »

16. Jean-Jacques Rousseau, "Ébauches», Confessions, in Euvres complètes, t. I, op. cit., p. 1155: «Les liaisons que j'ai eues avec plusieurs personnes me forcent d'en parler aussi librement que de moi. Je ne puis me faire bien connaître que je ne les fasse connaître aussi, et l'on ne doit pas s'attendre que dissimulant dans cette occasion ce qui ne peut être tu sans nuire aux vérités que je dois dire, j'aurai pour d'autres des ménagements que je n'ai pas pour moi-même. »

17. Jean Starobinski, La Relation critique, Paris, Gallimard, coll. « Tel », 2001, p. 117.

18. Jean-Jacques Rousseau, Les Rêveries du promeneur solitaire, in Euvres complètes, t. I, op. cit., p. 995; cf. ébauches des Confessions, ibid., p. 1150 : «Qu'on n'objecte pas que n'étant qu'un homme du peuple, je n'ai rien à dire qui mérite l'attention des lecteurs. Cela peut être vrai des événements de ma vie: mais j'écris moins l'histoire de ces événements en eux-mêmes que celle de l'état de mon âme, à mesure qu'ils sont arrivés. Or les âmes ne sont ni plus ou moins illustres que selon qu'elles ont des sentiments plus ou moins grands et nobles, des idées plus ou moins vives et nombreuses. [...] Dans quelque obscurité que j'ai pu vivre, si j'ai pensé plus et mieux que les Rois, l'histoire de mon âme est plus intéressante que celle des leurs. »

19. Antoine Lilti, Le Monde des salons. Sociabilité et mondanité à Paris au XVIII siècle, Paris, Fayard, 2005, p. 204 et 412.

20. Dans l'un de ses premiers essais sur les progrès des arts, Hume écrit: "Parmi les arts de la conversation, aucun n'est plus plaisant que cette déférence, cette civilité réciproque qui nous conduit à renoncer à nos inclinations personnelles pour celles de nos compagnons. [...] Quand le pouvoir s'élève du peuple jusqu'aux grands, comme dans toutes les républiques, de tels raffinements de civilité sont peu susceptibles d'être pratiqués puisque tout est, dans cette forme de gouvernement, presque au même niveau, et que les membres sont, dans une certaine mesure, indépendants les uns des autres. [...] Mais, dans une monarchie civilisée, il y a une longue chaîne de dépendance qui va du prince au paysan [...] qui est suffisante pour faire naître en chacun une inclination à plaire à ses supérieurs et à se former sur les modèles les plus reçus chez les gens de condition et d'éducation » («Essai sur la naissance et les progrès des arts et des sciences», 1742).

21. Arnault Skornicki, L'Économiste, la Cour et la Patrie. L'économie politique dans la France des Lumières, Paris, CNRS Éditions, 2011, p. 138.

22. Voltaire, Lettres philosophiques: «Entrez dans la Bourse de Londres, cette place plus respectable que bien des cours; vous y voyez rassemblés les députés de toutes les nations pour l'utilité des hommes. Là, le juif, le mahométan et le chrétien traitent l'un avec l'autre comme s'ils étaient de la même religion, et ne donnent le nom qu'à ceux qui font banqueroute » ( $6^{\mathrm{e}}$ lettre).

23. Je m'appuie ici sur Silvia Sebastiani, The Scottish Enlightenment. Race, Gender and the Limits of Progress, New York, Palgrave Macmillan, 2013.

24. Voltaire, «Seconde Épître dédicatoire à M. le chevalier Falkener, ambassadeur d'Angleterre à la porte ottomane », introduction à Zaïre, 1736.

25. David Hume, «Essai sur la naissance et le progrès des sciences et des arts » (1742), traduit par Philippe Folliot, en ligne sur Les classiques des sciences sociales: http://classiques.uqac.ca/ classiques/Hume_david/essai_sur_naissance_progres_arts/essai_naissance_progres_arts.html.

26. Jean-Jacques Rousseau, Lettre à d'Alembert, Paris, Garnier-Flammarion, 2003, p. 155; et p. 160 : «Nos cercles conservent encore parmi nous quelque image des mœurs antiques. Les hommes entre eux dispensés de rabaisser leurs idées à la portée des femmes et d'habiller galamment la raison, peuvent se livrer à des discours graves et sérieux sans crainte du ridicule. [...] Si le tour de la conversation devient moins poli, les raisons prennent plus de poids : on ne se paie point de plaisanterie, ni de gentillesse. On ne se tire point d'affaire par de bons mots [...]. S'il se mêle à tout cela quelque propos licencieux, il ne faut point trop s'en effaroucher: les moins grossiers ne sont pas 


\section{Philippe Roussin}

toujours les plus honnêtes. » Dans sa Défense des droits des femmes (1792), Mary Wollstonecraft cite ce passage du livre V de l'Émile: «L'homme dit ce qu'il sait, et la femme dit ce qui plaît; l'un pour parler a besoin de connaissance, et l'autre de goût [...] elles doivent s'imposer pour loi de ne jamais rien dire que d'agréable à ceux à qui elles parlent» (Paris, Gallimard, coll. «Folio», 2016, p. 128).

27. Nicolas Boileau, Satire, I: "Mais moi, vivre à Paris! Eh! qu'y viendrais-je faire? / Je ne sais ni tromper, ni feindre, ni mentir, / [...] Je suis rustique et fier, et j'ai l'âme grossière / Je ne puis rien nommer, si ce n'est par son nom, / [...] À quoi bon ces grands mots? doucement, je vous prie : / Ou bien montez en chaire, et là, comme un docteur, / Allez de vos sermons endormir l'auditeur: / C'est là que bien ou mal on a droit de tout dire. »

28. Nicolas-Toussaint Le Moyne Des Essarts, Causes célèbres curieuses et intéressantes, de toutes les cours souveraines du royaume, 1785, p. 53.

29. Philippe Raynaud, La Politesse des Lumières, op. cit., p. 178.

30. Robert Darnton, De la censure, Paris, Gallimard, coll. «NRF Essais », 2014, p. 14.

31. Charles Walton, Policing Public Opinion in the French Revolution, Oxford, Oxford University Press, 2009.

32. Sur les permissions tacites, voir Robert Darnton, De la censure, op. cit., p. 33-34.

33. Voir Rosario Villari, «Éloge de la dissimulation», et Jean-Pierre Cavaillé, «Pour une histoire de la dissimulation », Les Dossiers du Grihl, 2009-02, "Dissidence et dissimulation ».

34. Leo Strauss, Persecution and the Art of Writing, 1941.

35. Helvétius, De l'esprit (1758), classiques.uqac.ca/classiques, p. 479.

36. Ibid., p. 325.

37. Helvétius, De l'homme (1771), Londres, Société typographique, 1773 ; cité par Agnès Steuckardt, "Des mots en politique : Liberté de la presse, des Lumières aux Trois Glorieuses », Mots, $\mathrm{n}^{\mathrm{o}} 73$, «Les discours de la guerre », 2003, p. 173-182.

38. Helvétius, De l'esprit, op. cit., p. 21.

39. Emmanuel Kant, Que signifie s'orienter dans la pensée? (1786): "À la liberté de penser s'oppose premièrement la contrainte civile. Certes, on dit: la liberté de parler, ou d'écrire peut nous être retirée par un pouvoir supérieur mais absolument pas celle de penser. Toutefois, quelles seraient l'ampleur et la justesse de notre pensée, si nous ne pensions pas en quelque sorte en communauté avec d'autres à qui nous communiquerions nos pensées et qui nous communiqueraient les leurs! On peut donc dire que ce pouvoir extérieur qui dérobe aux hommes la liberté de communiquer en public leurs pensées, leur retire aussi la liberté de penser. »

40. Jean-Paul Marat, L'Ami du peuple, no 181, 8 avril 1790, p. 1 et 4.

41. Maximilien de Robespierre: "Le but essentiel de la liberté de la presse est de contenir l'ambition et le despotisme de ceux à qui le peuple a commis son autorité, en éveillant sans cesse son attention sur les atteintes qu'ils peuvent porter à ses droits » (Discours sur la liberté de la presse, prononcé à la Société des Amis de la Constitution, 11 mai 1791).

42. François Xavier Lanthenas, De la liberté indéfinie de la presse et de l'importance de ne soumettre la communication des pensées qu'à l'opinion publique, Paris, Imprimerie du patriote français, juin 1791, p. 26 et 37.

43. William Godwin, "Of Sincerity », in An Enquiry Concerning Political Justice, Oxford, Oxford University Press, 2013, p. 131 (ma traduction). Je renvoie à Timothy Michael, British Romanticism and the Critique of Political Reason, Baltimore, The Johns Hopkins University Press, 2016.

44. William Godwin, "Of Libels», in An Enquiry concerning Political Justice, op. cit., p. 341.

45. Donatien Alphonse François de Sade, Histoire de Juliette, in Euvres, Paris, Gallimard, coll. «Bibliothèque de la Pléiade», t. III, 1995, p. 143.

46. Ibid., p. 1261

47. Donatien Alphonse François de Sade, Idée sur les romans, Paris, Mille et Une Nuits, 2013. p. 28 ; Nicolas Edme Rétif de La Bretonne: "IXe Juvénale. Fausse immoralité de la liberté de la presse» (1796), Monsieur Nicolas, t. II, Gallimard, coll. «Bibliothèque de la Pléiade », 1989.

48. Joseph Pierre Chassan, avocat général près la Cour Royale de Colmar, Traité des délits et contraventions de la parole, de l'écriture et de la presse, t. I, Paris-Colmar, 1837, p. 2.

49. Émile de Girardin, Les 52, vol. X-XI, Le Droit de tout dire, Paris, Michel Lévy, coll. «Bibliothèque démocratique », 1849 , p. 6. L'auteur rappelle les propos auparavant tenus par Cavaignac devant la Chambre des députés en avril 1833: «Laissez s'établir par la presse le droit de tout dire 


\section{Tout dire ou le gouvernement de la langue}

envers et contre tous, elle en deviendra à la fois plus calme et plus explicite »; voir aussi, du même auteur, " Du droit de tout dire», in Les Droits de la pensée. Questions de Presse, 1830-1864, Paris, Michel Lévy, 1864, p. 322-350.

50. Charles Augustin Sainte-Beuve, à propos de Raphaël de Lamartine, Causeries du lundi, t. I. Paris, Garnier Frères, 1857, p. 67.

51. Victor Hugo, "Les Fleurs », in Euvres complètes, Critique, Paris, Robert Laffont, 1975. p. 535 et 543. Sur l'opacité sociale et la métaphore de la profondeur, voir Pierre Macherey, Que peut la littérature?, Paris, PUF, 1990, p. 77-125.

52. Cuvillier-Fleury, «Madame Bovary, par M. Gustave Flaubert», Journal des Débats, 26 mai 1857. À propos de ce nouvel idéal de l'explicitation exhaustive, voir G. Steiner, «Eros and Idiom », dans On Difficulty and Other Essays, Oxford, Oxford University Press, 1972 , p.112-114.

53. Charles Baudelaire, Euvres complètes, t. I, Paris, Gallimard, coll. «Bibliothèque de la Pléiade», 1975, p. 1206-1209.

54. Émile Zola, "L'argent dans la littérature », in Le Roman expérimental, Paris, Charpentier, 1880, p. 190.

55. Émile Zola, «La République et la littérature», in ibid., p. 374-375.

56. Anne Simonin, Le Déshonneur dans la République. Une histoire de l'indignité, 1791-1958. Paris, Grasset, 2008, p. $658 \mathrm{sq}$.

57. Ibid., p. 667-668: «Le droit ne remet pas en question la responsabilité historique des écrivains théorisée par Jean-Paul Sartre et exemplifiée par les grands procès de la répression des faits de collaboration (Brasillach, Rebatet). »

58. Jean-Paul Sartre, Qu'est-ce que la littérature? (1948), Paris, Gallimard, coll. «Idées », 1976. p. 27-28.

59. Gisèle Sapiro, La Responsabilité de l'écrivain. Littérature, droit et morale en France (XIXeXXI siècle), Paris, Seuil, 2011.

60. Jean-Paul Sartre, Qu'est-ce que la littérature?, op. cit., p. 82 et 95.

61. Maurice Blanchot, La Part du feu, Paris, Gallimard, 1949, p. 194.

62. Ibid., p. 306.

63. Maurice Blanchot, «L'inconvenance majeure» (1965), in Donatien Alphonse François de Sade, Français, encore un effort si vous voulez être républicains, Paris, Pauvert, 1965; repris sous le titre «L'insurrection, la folie d'écrire », in L'Entretien infini, Paris, Gallimard, 1969, p. 323-342. Sur les lectures de Sade depuis 1945, voir Éric Marty, Pourquoi le XXe siècle a-t-il pris Sade au sérieux?, Paris, Seuil, 2011. Maurice Blanchot est alors un opposant résolu au général de Gaulle depuis le retour de ce dernier au pouvoir en 1958: c'est ainsi qu'il faut comprendre le « toujours nous vivons sous un Premier consul». Il est également l'un des auteurs du Manifeste des 121, titré Déclaration sur le droit à l'insoumission dans la guerre d'Algérie, publié le 6 septembre 1960, capital pour l'avenir de la gauche et de l'extrême gauche en France.

64. Maurice Blanchot, Lautréamont et Sade, Paris, Éditions de Minuit, 1949, p. 25.

65. Jean Bessière, Principes de la théorie littéraire, Paris, PUF, 2005, p. 145.

66. Georges Bataille, "L'homme souverain de Sade», in L'Érotisme, Paris, Éditions de Minuit, 1957, p. 184.

67. Roland Barthes: "Hors le meurtre, il n'y a qu'un trait que les libertins possèdent en propre et ne partagent jamais, sous quelque forme que ce soit : c'est la parole. Le maître est celui qui parle, qui dispose du langage dans son entier; l'objet est celui qui se tait, reste séparé, par une mutilation plus absolue que tous les supplices érotiques [...] Seule cette parole est libre, inventée, se confondant entièrement avec l'énergie du vice. Dans la cité sadienne, la parole est peut-être le seul privilège de caste qu'on ne puisse réduire» (Sade, Fourier, Loyola [1971], in CEuvres complètes, t. II, 1966-1975, Paris, Seuil, 1994, p. 1061-1062).

68. Michel Foucault, «La pensée du dehors » (1966), in Dits et Écrits (1954-1988), t. I, 1954 1969, Paris, Gallimard, 1994, p. 518-540.

69. Michel Foucault, «La folie, l'absence d'œuvre» (1964), in ibid., p. 419. Vincent Descombes a dit la dimension politique à l'horizon d'une telle revendication. Celui qui prétend pouvoir tout dire signifie qu'aucune parole n'a véritablement valeur de loi, que la loi n'est qu'une convention, que l'interdit dépend d'un arbitraire et que rien, donc, n'interdit vraiment de parler. C'est moins le droit de parler que le pouvoir de tout dire qui est ici revendiqué, invitation à « me passer de la permission 


\section{Philippe Roussin}

des autres » et à "m'autoriser moi-même à me soustraire au joug symbolique », mais aussi à établir sur les autres le pouvoir de ma parole; voir Vincent Descombes, L'Inconscient malgré lui, Paris, Éditions de Minuit, 1977, p. 28 et 106 sq.

70. Jacques Derrida, Passions, Paris, Galilée, 1993, p. 64-67.

71. En référence à Sade sous les plumes de M. Vargas Llosa ou Philippe Sollers: «Le droit de la littérature à tout dire », un dialogue entre Mario Vargas Llosa, Gabriel Liiceanu, Dilema Veche, $\mathrm{n}^{\circ}$ 89, 30 septembre-6 octobre 2005: http://psychanalyse-paris.com/Le-droit-de-la-littérature-atout.html; Philippe Sollers, Jacques Soulillou, «L'art peut-il tout dire? », Le Monde des débats, juillet-août 1999. Voir aussi Le Monde/Villa Gillet, Le Roman: tout dire? Assises du roman 2010. Paris, Christian Bourgois, 2010.

72. Georges Perec, La Vie mode d'emploi (1978), Paris, Le Livre de Poche, 2010, p. 152.

73. Roland Barthes par Roland Barthes, Paris, Seuil, 1975, p. 182.

74. Ibid., p. 122.

75. Roland Barthes, Sade, Fourier, Loyola, op. cit., p. 1131.

76. Gustave Flaubert, lettre à Louise Colet du 16 décembre 1852, Correspondance, t. II, 1851 1858, Paris, Gallimard, coll. «Bibliothèque de la Pléiade », 1980, p. 208-209.

77. Prétexte: Roland Barthes / Cerisy 1977, Paris, Christian Bourgois, 2003, p. 26.

78. Ibid., p. 24-25.

79. Roland Barthes, » Saussure, le signe, la démocratie» (1973), in Euvres complètes, t. II, op. cit., p. 1585-1587.

80. Sur l'importance égale de la parrhêsia et de l'isègoria dans la démocratie grecque, voir Ineke Sluiter et Ralph. M. Rosen (eds), Free Speech in Classical Antiquity, Leiden, Brill, 2004.

\section{RÉSUMÉ}

Tout dire ou le gouvernement de la langue

Tout dire est une expression qui apparaît dans la langue française au XIII ${ }^{\mathrm{e}}$ siècle. Les contextes d'usage et les inflexions sémantiques qu'elle a connues permettent de comprendre les modifications successives des frontières entre sphère privée et sphère publique. On analyse la période 1750-1990 en isolant trois moments : naissance de l'autobiographie et droit de la critique, jusqu'à la période révolutionnaire au XVIII ${ }^{\mathrm{e}}$ siècle; combats autour de la liberté de la presse et de l'esthétique réaliste au XIXe siècle; débats concernant la place de la littérature à partir de 1945 et jusque dans les années 1960, où l'expression en vient à désigner ce qui semble être la tâche de la littérature.

\section{SUMMARY}

\section{Saying everything or the government of the tongue}

To say everything is an expression that appears in the French language in the 13th century. The contexts of use and semantic inflections it has known allow an understanding of the fluctuating boundaries between private and public spheres. An analysis of the 1750-1990 period isolates three moments : the birth of autobiography and the right to criticize, until the revolutionary period in the 18th century; fights around the freedom of the press and realistic aesthetics in the 19th century; debates about the place of literature from 1945 until the 1960 s when the term comes to mean what appears to be the aim of literature. 
Tout dire ou le gouvernement de la langue

\author{
RESUMEN
}

Decir todo o el gobierno del lenguaje

Decir todo es una expresión que aparece en la lengua francesa del siglo XVIII. Los contextos de uso y las inflexiones semánticas que ha conocido permiten comprender las modificaciones sucesivas de las fronteras entre la esfera privada y la esfera pública. Se analiza el periodo 1750-1990 escogiendo tres momentos : nacimiento de la autobiografía y derecho de la crítica, hasta el periodo revolucionario en el siglo XVIII; combates en torno a la libertad de prensa y la estética realista en el siglo XIX; debates relacionados con el lugar de la literatura a partir de 1945 y hasta los años 1960, donde la expresión designará lo que parece ser la tarea de la literatura. 